\title{
PráCticA HISTORIOGRÁFICA Y CIENCIAS SOCIALES EN MÉXICO. UnA APROXIMACIÓN A LA HISTORIA CONTEMPORÁNEA Y DEL PRESENTE
}

\section{Historiographic Practice and Social Sciences in Mexico. An Approach to Contemporary and Present History}

\author{
Martín López-Ávalos* \\ DOI: http://dx.doi.org/10.29043/liminar.vl8il.733
}

Resumen: Se abordan las condiciones que explican la evolución de la práctica disciplinaria de la historia en México a lo largo del siglo XX. Esta revisión permite advertir que dicha práctica es el resultado de un contexto político, nacional e internacional, y de procesos históricos que definimos como la contemporaneidad. Esta, a su vez, refleja la forma en que las disciplinas abocadas a su estudio se despliegan perfilando una colaboración inter y transdisciplinaria (hibridación). Este proceso se observa con el surgimiento, en primer lugar, de subdisciplinas incorporadas a la práctica historiográfica — historia económica, social, demográfica, por ejemplo, o sociología histórica-y, en segundo lugar, de campos temáticos que se relacionan con la historia del presente como la etapa más novedosa. Se termina haciendo un recuento de la práctica de esta en México en las últimas décadas.

Palabras clave: historia contemporánea; historia del tiempo presente; ciencias sociales; interdisciplinariedad.

Abstract: The conditions behind the evolution of the study of history in Mexico throughout the 20th century are addressed. This review discusses the fact that the study of history is the result of a political, national, and international context, of historical processes that we define as a contemporaneity. This, in turn, reflects the way in which the disciplines within this study unfold in an interdisciplinary and transdisciplinary collaboration (hybridization). This process can be observed first with the emergence of sub-disciplines incorporated into historiographic practice - economic, social, demographic history, for example, or historical sociology — and then with the thematic fields that relate to current history as the newest stage. The review concludes by outlining how this practice has been undertaken in Mexico in recent decades.

Keywords: contemporary history; history of the present time; social sciences; interdisciplinary.

\footnotetext{
* Martín López Ávalos. Doctor en Estudios Latinoamericanos por la Universidad Nacional Autónoma de México. Profesor investigador titular C de tiempo completo en el Centro de Estudios Históricos de El Colegio de Michoacán, México. Temas de especialización: historia política de América Latina en el siglo XX, vanguardias y movimientos insurreccionales; procesos de interpretación para la historia del tiempo presente. Correo electrónico: mlopez@colmich.edu.mx. ORCID: https:// orcid.org/0000-0002-1774-5258
}

Enviado a dictamen: 22 de marzo de 2019. Aprobación: 22 de mayo de 2019.

Revisiones: 2. 


\section{Introducción}

$\Lambda$ 1 presentar América Latina. Historia de medio siglo, Pablo González Casanova (1977) anunciaba la obra como un trabajo pionero en la producción historiográfica mexicana del momento, pues se trataba de una "historia actual" que marcaría el rumbo en el desarrollo de los estudios de historia contemporánea, y vaticinaba que su aparición alentaría a otros historiadores a producir historias de este tipo. Para González Casanova, lo "actual" no se refería a los años cercanos ni a la novedad del tema, sino más bien a la idea de explicar el tiempo presente a partir de la historia contemporánea. En un contexto disciplinario dominado por la práctica documental en torno al pasado, la propuesta de la historia de medio siglo resultaba innovadora, pues la misma se debía a la comparación en un estudio general de América Latina pero sin perder la especificidad de cada caso. En ella, se establece el esfuerzo por hacer un nuevo tipo de historiografía en la que se concilien los objetivos y tareas disciplinarias entre la historia y las ciencias sociales, ya que:

Por lo común los historiadores no se ocupan de la historia inmediata. Los sociólogos y politólogos, tampoco. Unos se quedan tradicionalmente en el pasado lejano. Otros, consideran que su tarea no es la del historiador. El vacío ha quedado en parte cubierto. Y será cubierto cada vez más en los próximos años (González Casanova, 1977:VII).

Constituye, además, una historia que, sin dejar de ser nacional, conforme se desarrolle será cada vez más latinoamericana e internacional (González Casanova, 1977:VII). El fomento de este tipo de estudios se extendió en los siguientes años; aparecieron colecciones y nuevos títulos dedicados a la historia inmediata como vaticinaba González Casanova en su presentación, sin embargo, y a diferencia de la experiencia europea, el esfuerzo sistemático no desembocó en la creación de un centro de investigación específico en historia inmediata o contemporánea. Con todo ello, González Casanova se inscribió en un debate en torno a la naturaleza de la relación entre la historiografía y el conjunto de las ciencias sociales, primero como historia contemporánea, para plantear varias décadas después otro terreno, el de la historia del presente, como había sucedido en los principales países europeos luego de la Segunda Guerra Mundial. El asunto no resulta menor si nos percatamos de que por los mismos años en Francia se institucionalizó una nueva práctica historiográfica derivada de la historia contemporánea que se denominó Historie du Temps Présent, mientras que en Alemania la Zeitgeschichte y en Inglaterra la Contemporary History se mantenían como conceptos para referirse al mismo fenómeno con establecimientos específicos para una práctica diferenciada del resto de la historiografía (Navajas, 2003:150).

A poco más de cuarenta años de la aparición de América Latina. Historia de medio siglo, es necesario preguntarnos por el destino que corrió la propuesta de hacer una historia diferente a la que imperaba en esos años en México, no solo para la historiografía sino también para las ciencias sociales y la relación entre ambas, como convocaba González Casanova. En este contexto, en el presente ensayo se pretende hacer una revisión de las condiciones que explican la evolución de la práctica disciplinaria de la historia para, desde ese marco, vincular su desarrollo con las ciencias sociales, primero como historia contemporánea, y luego como historia del tiempo presente. Así, nuestro abanico temporal parte de la década de 1940 en adelante, al mismo tiempo que se hace referencia a las condiciones previas que, a su vez, explican la lógica de la práctica disciplinaria de la historia. Esta revisión nos permitirá advertir que dicha práctica es el resultado de un contexto político, nacional e internacional, de procesos históricos que definimos en un periodo específico, el de la contemporaneidad que, a su vez, refleja la forma en que las disciplinas abocadas a su estudio se despliegan perfilando una colaboración inter y transdisciplinaria. Este proceso de hibridación lo podemos observar en primer lugar con el surgimiento de subdisciplinas incorporadas a la práctica historiográfica -historia económica, social, demográfica, por ejemplo, o sociología histórica - y en segundo lugar con los 
campos temáticos que se relacionan con la historia del presente como la etapa más novedosa.

El presente ensayo no procura abarcar la discusión disciplinaria dentro de la historiografía en torno a la legitimidad de la historia del presente, que generalmente se enfoca en problemas de métodos y fuentes, y en un aspecto más teórico, en la construcción del tiempo histórico de un pasado cerrado e inmóvil a otro abierto e inacabado. Lo que sí hacemos es tratar de mostrar que la práctica disciplinaria de la historia en México se ha hecho cada vez más heterogénea, diversa y plural con la incorporación de una comunidad, igual de heterogénea, cuyos integrantes se reconocen como practicantes de la historia del presente en espacios institucionales como las universidades, institutos y centros de investigación. En este esfuerzo confluyen no solo historiadores, sino también profesionales formados en alguna de las disciplinas sociales, con lo que el llamado a llenar huecos, como pidió González Casanova, sin duda ha sido atendido.

\section{Institucionalización y profesionalización}

La tradición historiográfica profesional en México es relativamente corta o al menos así lo ha hecho parecer la historia de la historiografía mexicana al anclar su inicio en la década de 1940, cuando la institucionalización del sistema universitario mexicano era un hecho consumado (Zermeño, 1997 y 2013). Si bien esto es cierto para explicar una de las etapas de este proceso, se deja de lado la disputa previa que hizo posible generar el primer espacio de discusión historiográfica que no demandó de un sistema educativo superior que le diera soporte y legitimación. Este espacio es un producto del liberalismo triunfante de la restauración anticolonialista a la derrota del Imperio de Maximiliano y la intervención francesa. Su gestación tardará una generación para ofrecerse como doctrina madura y acabada para el centenario del Estado mexicano en 1910: el positivismo.

El positivismo se convierte en teoría para el Estado antes que en ideología de este; con él, se construye una teoría de la historia que crea la narrativa historiográfica mexicana con sus periodos antiguo, medio y moderno. Tal es su impacto, que moldea la historiografía como una continuación del debate político de la época, primero entre liberalismo versus conservadurismo y luego como hegemonía liberal a lo largo de la República restaurada y buena parte del porfirismo, cuando encadena su destino al régimen político. Es precisamente en esta circunstancia eminentemente política donde se realiza el debate de la pertinencia de la historiografía como disciplina científica. Sobresalen dos aristas: el método y la teoría; con el primero aparece el empirismo como única prueba de trabajo científico no especulativo; con el otro, la idea de ciencia como paradigma que explica la sociedad moderna. Si la historia no es filosofía por especulativa, tampoco es su negación, porque aspira a encontrar una razón instrumental para conocer las leyes que gobiernan el universo físico y social, es decir, humano (Matute, 1991). El positivismo, ahora convertido en ideología del Estado, llega al final de su ciclo con un modelo educativo con el que aspiraba a coronar una universidad como cúspide de la educación nacional; con esta ideología se plantea la creación de una institucionalidad en la que las disciplinas científicas, acorde con la práctica de este canon, tuvieran cabida. Su desarrollo corre paralelo a la consolidación del sistema de educación superior en México, primero con la creación de la Universidad Nacional en 1910, y junto a ella la Escuela de Altos Estudios, y su readecuación como institución autónoma en 1929, así como la creación de un sistema de universidades públicas autónomas en los estados de la República, herederas de los institutos científicos y literarios fundados por los liberales en el siglo XIX, y de la transición del Museo Nacional al Instituto Nacional de Antropología e Historia (INAH), donde se considera la formación de profesionales de la historiografía, la arqueología y la antropología como parte de sus funciones. Con la Escuela de Altos Estudios se establece el origen de la primera experiencia de profesionalización disciplinaria en el país. Al amparo del Estado, la universidad debe propiciar las condiciones de reproducción del conocimiento disciplinario, si bien 
bajo un paradigma específico, de las ciencias positivas en cuyo paraguas se encuentran las ciencias sociales y la historiografía, es indudable que esta experiencia proporciona condiciones que una generación después contribuyen al segundo proceso de profesionalización científica de las disciplinas universitarias a partir de la década de 1940. El proyecto, publicitado como ejemplo de madurez del Estado nacional mexicano, pasa por la crítica de las armas del nuevo liberalismo que enarbola el modelo del cambio a través de su instrumento predilecto, la Revolución.

El conjunto de acontecimientos que llamamos Revolución mexicana hace tabla rasa del modelo anterior, el porfirista, incluso en su proyecto educativo coronado con la Escuela de Altos Estudios, cuando logra establecer un nuevo régimen político. La Revolución interrumpe, en cierto sentido, el camino de la institucionalización de las disciplinas científicas en su totalidad hasta nuevo aviso, cuando vuelve a plantearse la necesidad del conocimiento científico para el proyecto emanado del Estado en busca de nuevas definiciones de lo nacional en un mundo en transformación tras el colapso de las monarquías absolutistas derrotadas en la Primera Guerra Mundial. La Revolución mexicana aparece, entonces, como el presente contemporáneo que permite explicar la historia nacional en sus tres ciclos esenciales: prehispánico, novohispano y republicano, y al mismo tiempo dar parte del presente como resultado de la Revolución. A este contexto se debe la formación de los profesionales de la historia y las ciencias sociales en México desde sus inicios, al igual que su evolución futura en lo que resta del siglo XX. En buena medida, la tradición historiográfica y de ciencias sociales mexicanas se relaciona con el presente contemporáneo, incluso en su polo negativo como reacción en contra de él y su refugio en el pasado novohispano y prehispánico como prácticas disciplinarias. El proyecto nacional del Estado posrevolucionario mexicano requirió, entonces, de la participación de los profesionales de la historia y de las ciencias sociales formados en el sistema de educación superior para darle forma al presente como realidad palpable de la Revolución. Esta unión entre la historiografía y las ciencias sociales en buena medida es producto de una razón instrumental política del Estado contemporáneo mexicano. No resulta extraño comprobar que ha sido la necesidad de justificar la existencia del Estado nacional, desde su fundación en el siglo XIX, sus posteriores etapas en el siglo XX y los años que van del actual siglo, la que marca el acento de las discusiones de la academia mexicana. El tono de urgencia política está presente en cada uno de los debates. Por ejemplo, la producción de las historias generales, vale decir contemporáneas, de $\mathrm{México}^{1}$ en las dos últimas décadas muestran esta característica, además de exhibir la reunión de diversas disciplinas sociales junto con la historiografía. Véase el volumen coordinado por Cordera (2010) dentro de Historia crítica de las modernizaciones en México, obra escrita por un grupo amplio de académicos de diversos orígenes institucionales y disciplinarios, justificados en buscar "perspectivas actuales para ubicar las experiencias de cambio y modernización que ha vivido este país", dicen los editores en la presentación de la obra, para trazar caminos hacia el futuro, como afirman: "Solo así se podrá hacer una revisión racional que permita trazar trayectorias futuras más promisorias" (Cordera, 2010). Otro ejemplo lo aportan Bizberg y Meyer (2003), quienes ponderan la transición mexicana a partir de la crisis de 1968 como paradigma de los límites del modelo político posrevolucionario, su transición a uno nuevo y los avatares del mismo. La idea del cambio predomina en el análisis, al mismo tiempo que busca tanto iluminar el presente como advertir un futuro. Como puede observarse, el presente es definido como cambio y transformación que requiere del concurso de la historiografía y las ciencias sociales para captarlo.

El vínculo con los sucesos externos es otra de las características de la nueva época histórica no solo por existir un apremio por saber el lugar que guardan las sociedades emergentes, sino también por las relaciones internacionales que van planteando la idea de una comunidad internacional de Estados soberanos primero con la Sociedad de las Naciones y posteriormente con la Organización de las Naciones Unidas (ONU); en ambos casos aparecerán modelos de colaboración que impactan en diversos ámbitos como el de la educación 
superior. Este contexto es el que renueva el modelo de pertinencia de las prácticas diferenciadas de las disciplinas científicas y sociales y otorga, nuevamente, a la universidad el privilegio de convertirse en el "lugar" canonizado para producir y reproducir el conocimiento científico (Wallerstein, 1996).

Con la aparición de organismos internacionales derivados del funcionamiento de una comunidad internacional de naciones cuyo frente es la ONU pueden observarse los contornos de una nueva etapa de contemporaneidad que, más allá de los dilemas concretos de la realpolitik de la guerra fría y sus múltiples aristas, aspira a construir nuevas relaciones basadas en la cooperación entre naciones. Este contexto internacional proporciona nuevos instrumentos de cooperación, identificando la educación y las disciplinas académicas como agentes de cambio que requieren espacios de participación propicios para la nueva tarea. El surgimiento de organismos derivados de la ONU, como el dedicado a la educación, la ciencia y la cultura (UNESCO), abre nuevos horizontes para las disciplinas de lo social en la medida en que señalan una nueva ruta de acción que vincula tanto los paradigmas y teorías con las prácticas específicas de las disciplinas a través de la interdisciplinariedad, como un método de integración y cooperación del conocimiento, al mismo tiempo que recomienda la enseñanza de la historia contemporánea como un medio de entendimiento entre la comunidad de naciones.

La UNESCO, la Comisión Económica para América Latina (CEPAL) y el Instituto Panamericano de Geografía e Historia (IPGH) son los organismos más influyentes en la construcción del nuevo horizonte de expectativas disciplinarias, pues por un lado animan a la revisión de los contenidos curriculares de la educación profesional de la historiografía y de cada una de las disciplinas sociales con el objetivo de señalar la importancia de vincular el estudio de la historia contemporánea con el desarrollo económico y la integración de lo social (Tavera, 1955:624-636; Zavala, 1952:11, 14), y por otro estimulan la creación de instituciones educativas con un perfil acorde con estos objetivos, tal como la Facultad Latinoamericana de Ciencias Sociales (FLACSO) creada en 1957 como resultado de la Primera Conferencia Regional sobre la Enseñanza Universitaria de América del Sur celebrada en Río de Janeiro un año antes. No está de más señalar que incluso la universidad como tal es motivo de esta revisión a partir de modelos de educación que son generados en instancias similares como la Asociación Internacional de Universidades. Nos encontramos ante un movimiento internacional que involucra diversos niveles de participación: de la unidad disciplinaria donde se imparte la educación profesional, a las instituciones de educación superior que participan en asociaciones internacionales, incluidas las nacionales como la Asociación Nacional de Universidades e Instituciones de Educación Superior (ANUIES) y regionales como la Unión de Universidades de América Latina (UDUAL). Todo este movimiento recala en las mutaciones de los paradigmas teóricos de las disciplinas y en las prácticas específicas, sin las cuales los conceptos de interdisciplinariedad y cooperación para el desarrollo carecen de sentido (Wallerstein, 1996; Iggers, 2012).

\section{Espacios de colaboración}

En el ámbito disciplinario de la historiografía, una vez institucionalizado su espacio de desarrollo en las universidades y centros de investigación de la década de 1940 en adelante, el cambio fundamental lo observamos en el quiebre del paradigma de la historia cientificista asociada con el positivismo, que consideraba la historia como el estudio del pasado, razón por la cual el historiador profesional rara vez traspasaba esa frontera en la primera etapa de profesionalización en México. A partir de entonces, la historiografía se nutrirá de las técnicas y métodos emanados de las ciencias sociales; aparecerán las subdisciplinas como las diversas variedades de historiografías asociadas con una ciencia social específica: económica, social, demográfica y un sinfín de variedades que se derivan de estas, como historia de la industrialización, historia urbana, etcétera (Gortari, 1990 y 2002). Sin embargo, la ruptura con esa frontera imaginaria aludida la darán los historicistas del medio siglo — la década de 1950-, 
cuando postulan el cambio del criterio de verdad historiográfica mantenido por la escuela positivista. Lo contemporáneo es importante no como tema o periodo de estudio, sino como contexto desde donde se escribe, pues es el presente el que interroga al pasado, por lo cual cualquier acto de comprensión histórica es un acto del historiador al momento de escribir, como un acto o hecho del presente (Zermeño, 2013). La influencia de la escuela historicista mexicana matizará la relación del pasado con el presente en buena medida, en relación con la forma en como los historiadores fueron aceptando la contemporaneidad como un periodo historiográfico que podía ser atendido con los mismos criterios de rigor documental con que se estudiaba el pasado. La certeza de verdad construida por el respaldo documental sería poco a poco modificada al hacerse presente, en la misma medida, la subjetividad del historiador en buena parte del uso de sus técnicas y métodos, para culminar con la interpretación de los datos derivados de los documentos (Gortari, 2002).

La producción historiográfica inicia una nueva etapa en varios sentidos. El primero y más obvio es la legitimidad de la historia contemporánea como una práctica disciplinaria a la par de cualquier otra, con el añadido de que en su espacio es factible la integración con cualquiera de las disciplinas sociales, como venían señalando las recomendaciones a la educación superior derivadas de los estudios y diagnósticos apadrinados por la UNESCO. Otra dimensión importante fue el triunfo de la visión historicista, según la cual toda escritura histórica es un acto del presente y, como tal, es necesario conocerlo. A esto habrá que añadir las demandas de nuevo conocimiento surgidas en los nuevos tiempos que corrían con la posguerra - 19451989-, el más importante, el problema del desarrollo de los países emergentes, como México y los de América Latina, pero también los que salían del dominio colonial en Asia y África, sin minimizar el gran cambio social y cultural que se produjo en los países industrializados de ese momento. Al transformarse el contexto, nacional e internacional, que produce el conocimiento social, cambiaron las circunstancias, como dirían los historicistas, y por lo tanto las preguntas, temas y periodos que se requiere conocer: surgen nuevos sujetos y problemas que no estaban visibles en el periodo anterior y que hacen reconocible nuestro tiempo.

Si bien el espacio de colaboración e integración entre el conocimiento historiográfico y el de las ciencias sociales es previo, como ya señalamos, la novedad es que ahora, sin acudir a las subdisciplinas historiográficas, es posible contar con un lenguaje conceptual común para plantear nuevos problemas e interpretaciones. Al hacer un balance del periodo, un historiador consideraba que: "La historiografía política tradicional no parecía suficiente para abarcarlos y estudiarlos, por lo que surgió la pregunta: ¿cómo estudiarlos en el pasado? La renovación cobraba sentido" (Gortari, 2002:31). La renovación señalada se justificaba por su atención al presente, a los problemas derivados de la nueva época que requerían de una revisión histórica. Con ello, la historiografía a partir de la década de 1970, y sobre todo posterior a la década de 1990, amplió sus campos de estudio sin dejar de reconocerse en un centro o núcleo disciplinario que abarca el pasado, el presente y el futuro. La nueva dinámica amplió los márgenes con nuevas prácticas al permitir incorporar de manera flexible temas, métodosy, como novedad, nuevas fuentes de información, así como su manejo, almacenamiento y procesamiento. Desde el punto de vista de la práctica que se considera, todavía, el núcleo de la disciplina, esta dinámica es percibida como la especialización al infinito que produce superespecialidades que se dividen una de otra. Si observamos el asunto desde otro punto, fuera del núcleo disciplinario, desde los márgenes, la idea disciplinaria de la historia está construida por un conjunto de prácticas — muchas de ellas ni siquiera derivadas de las subdisciplinas historiográficas establecidas- producidas por las ciencias sociales en su propio camino de adaptación a los cambios de tiempos contemporáneos (Trindade, 2007).

En este contexto de hibridación o mutación permanente del trabajo historiográfico fue donde surgió la historia del tiempo presente. El debate internacional en torno a la historia del tiempo presente ha calado de diferentes maneras en México. Sin duda la influencia de las escuelas europeas no pasó en balde, sobre todo 
la francesa y la inglesa (Aróstegui, 1998 y 2004; Soto, 2004; Cuesta, 1983). Si bien la introducción de la distinción entre historia contemporánea e historia del tiempo presente puede explicarse por la influencia de dichas escuelas en la comunidad historiográfica mexicana, su aparición también coincide, como señalamos anteriormente, con el fin de la necesidad del Estado nacional por justificar su existencia a partir de la experiencia revolucionaria de 1910. La aparición de nuevas narrativas que cuestionan este canon es el signo más visible de los nuevos aires que tocan a la historiografía mexicana en general, y en particular a la que atañe al periodo contemporáneo; no se trata, sin embargo, de plantear un nuevo paradigma como se viene desarrollando en Francia con el Instituto de Historia del Tiempo Presente (Bédarida, 1998; Sauvage, 1998) y la historiografía específica que va produciendo. En México, su particularidad está en los tipos de práctica historiográfica que se presentan de manera diferenciada. Aparecen, por un lado, la historia oral (Garay, 2007) como un ejercicio específico disciplinario más cercano a la historia del tiempo presente y, por otro, la investigación de interpretación del proceso contemporáneo -mexicano y latinoamericano-, más involucrado con un tipo específico de ciencias sociales volcado al conocimiento como acción política para la transformación social, como lo ha planteado González Casanova en todos los proyectos académicos que ha venido animando desde la década de 1970.

\section{La práctica y la institucionalización: la historia del presente}

Como puede observarse, en México existen y conviven prácticas diferenciadas que comparten un periodo historiográfico, entendido como historia contemporánea más que como historia inmediata o del presente. Sin embargo, esto no implica, necesariamente, compartir un método o paradigmas epistemológicos en relación con la definición del tiempo histórico del presente, como sucede en el caso francés. Esa discusión o debate no ha llegado a la comunidad interesada en este tema, llámese historiadores o científicos sociales. El caso mexicano se caracteriza por operar sin la necesidad de un marco institucional específico, llámese instituto o centro de estudios en historia contemporánea o del tiempo presente. ${ }^{2}$ Esto se puede explicar porque la estructura establecida en la génesis de su profesionalización — década de 1940 - había dado cabida a las diversas prácticas específicas de los estudios contemporáneos en los institutos de investigaciones históricas y sociales y en la docencia a través de la licenciatura y el doctorado en historia en la Facultad de Filosofía y Letras, y en la Escuela Nacional de Ciencias Políticas y Sociales de Universidad Nacional Autónoma de México (UNAM), donde se impartían sociología y ciencia política. ${ }^{3}$ Así, por ejemplo, el Instituto de Investigaciones Históricas de la UNAM, creado en 1944, cuenta con un área de investigación para la historia moderna y contemporánea, y desde 1965 con una revista especializada en estos temas, Estudios de Historia Moderna y Contemporánea, que como resulta obvio no está dedicada al presente pero sí mantiene un vínculo con estos temas, tanto por la actividad de sus autores como por los artículos publicados en ella. La Facultad de Filosofía y Letras, por su parte, es la instancia encargada de la docencia y formación de profesionales de la historia en la licenciatura y el posgrado, y cuenta con una tradición, por lo menos desde 1953 en adelante, de vincular el currículo con la historia contemporánea y las ciencias sociales (Tavera, 1955:624-636). En la actualidad esta facultad alberga el Seminario de Historia Contemporánea y del Tiempo Presente, con lo cual es de esperarse que algunas de las tesis que elaboran los estudiantes de historia se acerquen a este campo no solo desde el tema o periodo, sino desde una metodología específica que se distinga de otras prácticas historiográficas. A diferencia del ámbito de investigación, en el que existen ejercicios regulares para establecer estos parámetros, la formación docente carece de estudios de este tipo, es decir, no existen estudios sistemáticos ni cuantitativos ni cualitativos que nos indiquen las variedades de tesis de licenciatura y posgrado con las que los alumnos de historia se gradúan como profesionales de licenciatura 
y doctorado. Este tipo de estudios permitiría establecer si existe un cambio en las preferencias de las nuevas generaciones hacia la historia contemporánea y del presente, dominado por el estudio del pasado colonial y republicano en su primer siglo. Este ejercicio tendría que abarcar toda la geografía institucional de la educación superior mexicana, pues si bien la UNAM sigue siendo un referente importante, en la actualidad existen programas de formación de historiadores en casi todas las instituciones, públicas y privadas, en licenciatura y posgrado.

Por su parte, el Instituto de Investigaciones Sociales de la UNAM, fundado en 1930, destaca por la amplitud de sus áreas de investigación para, siguiendo la tradición heredada de Andrés Molina Enríquez, contribuir a la solución de los problemas nacionales desde la óptica de las ciencias sociales. Dos cosas sobresalen en este esfuerzo para el tema que nos atañe. En primer lugar, la Revista Mexicana de Sociología, aparecida en 1939, donde se reflejan los cambios teóricos y empíricos de la sociología mexicana y su relación con la historia, ya sea como historiografía o como tiempo y espacio específicos relacionados con la sociedad mexicana. Es de destacar, nuevamente, el espacio dedicado a los temas contemporáneos de América Latina, con lo cual, como veremos con otras áreas, existe un vínculo — sea interdisciplinario o no- entre la teoría social y la historia contemporánea que linda con América Latina como referente y como constante con el tiempo presente. En segundo lugar, la creación de seminarios, el primero de México contemporáneo y el segundo de Historia del Tiempo Presente. Este último se manifiesta mucho más activo que el primero, pues a través de sus cuentas en las redes sociales ${ }^{4}$ muestra una actividad constante que va en aumento. Llama la atención, en este ejemplo, no solo la amplitud de perspectivas disciplinarias de la historia que asumen la práctica del tiempo presente, sino los campos temáticos específicos derivados de las ciencias sociales, tan amplios y variados como son las prácticas actuales de este conjunto de disciplinas. El grupo que integra este seminario, encabezado por una investigadora del instituto, ya organizó un coloquio donde se presentaron diversos trabajos que van desde la reflexión historiográfica sobre los límites y expectativas como práctica, a los estudios empíricos dedicados a la memoria o la violencia política - desaparecidos- en América Latina, como los temas predominantes.

El INAH, junto a otras instituciones de educación superior, ha participado de este esfuerzo a través del Departamento de Investigaciones Históricas, fundado en 1959 y transformado en Dirección de Estudios Históricos en 1979, como se mantiene hastala actualidad. Inicialmente dedicado a la historia prehispánica, a partir de la década de 1970 fue transformando el perfil institucional de la investigación, primero con la incorporación de seminarios hacia un abanico de temas más amplio de la historia que el periodo prehispánico, tocando aspectos sociales, culturales y económicos. Sin embargo, fue en la década de 1980 cuando se incorporó de lleno el interés por la historia contemporánea como un área específica de investigación. ${ }^{5}$ A partir de este núcleo, en 2014 apareció la primera revista disciplinaria, es decir, desde la historiografía, y con un formato digital, Con-Temporánea, ${ }^{6}$ dedicada a la historia muy contemporánea y del presente, como anuncian sus creadores y primeros editores: "Toda la historia en el presente" con la intención de asumir la historia inmediata, la del siglo XX recién concluido y la que ha transcurrido del presente siglo, como un trabajo colectivo que se va tejiendo en voces múltiples, a veces contradictorias y cambiantes, para "traer lo muy lejano en el tiempo-espacio al diálogo con este tiempo presente, distanciarse del presente sólido y confiar para abrirlo a las posibilidades múltiples y empujarlo" (INAH, 2014).

Pese a esta falta de formalizar un espacio institucional exclusivo para la historia contemporánea, inmediata o del presente, la práctica se ha establecido en diversas instancias de formación de posgrado - maestrías y doctorados- y de investigación como los ya mencionados institutos de investigaciones históricas y sociales de la UNAM, así como en otros de carácter interdisciplinario y temático dentro de la misma institución; por ejemplo, en 1986 se creó el Centro de Investigaciones Interdisciplinarias en 
Humanidades, ampliando su ámbito a las ciencias en 1995, como continúa hasta ahora bajo las siglas de CEIICH, con el propósito de tender puentes entre las disciplinas involucradas para atender problemas y realidades complejas de México y el mundo. ${ }^{7}$ La labor de investigación en este centro, por ejemplo, tiene una presencia significativa a través de un fondo editorial dedicado a los problemas del presente, "El Mundo del Siglo XXI", que refleja la labor de los investigadores pertenecientes al programa del mismo nombre. Esta colección ha publicado veinticinco títulos entre 1996 y 2004. Otras colecciones que se desprenden de la labor de investigación de este centro son "Las Ciencias y las Humanidades en los Umbrales del Siglo XXI", con un total de dieciocho títulos publicados entre 1997 y 2000, y "El Mundo Actual”, con seis títulos publicados entre 2004 y 2015. A través de este ejemplo, es evidente la existencia de una práctica en torno a temas de investigación y campos de conocimiento innovadores para la estructura disciplinaria de la historiografía mexicana, y sobre todo en la relación con las ciencias sociales vista como trabajos de síntesis en torno a problemas específicos. Los contenidos y periodos abordados en estas tres colecciones son variados y abarcan temas de reflexión metodológica de las ciencias sociales en México y América Latina. En este punto es importante resaltar la relación, tanto interdisciplinaria como temática y temporal, entre el espacio regional latinoamericano donde se desenvuelve la historia y los procesos sociales nacionales, sin ser el centro pero sí una constante; la variable latinoamericana es otro de los rasgos de las prácticas de este tipo que van perfilando las formas de trabajo interdisciplinario que aborda lo contemporáneo y del presente. La UNAM cuenta con un centro de investigaciones dedicado a América Latina, el Centro de Investigaciones sobre América Latina y el Caribe (CIALC), que ha pasado por una transformación, de ser un programa coordinador y difusor, a un espacio de investigación plena; al mismo tiempo, existe un programa docente de licenciatura y posgrado dedicado a esta área, lo cual muestra la existencia de una comunidad dedicada al estudio del espacio contemporáneo y del presente desde la perspectiva de la historia regional o los estudios de área. Esta característica demanda una mirada más detenida y pausada para integrar el mapa de los estudios del presente.

A las instituciones pioneras en el campo se les han ido sumando otras creadas, todavía, en el periodo de expansión del Estado mexicano; por la importancia de nuestro tema destacaremos el Instituto de Investigaciones Dr. José María Luis Mora, convertido en institución de investigación histórica en 1981, y con actividades de formación profesional con la maestría de historia moderna y contemporánea desde 1994, transformada en doctorado diez años después. La orientación hacia la historia contemporánea ha dado pie a un desarrollo de las líneas de investigación del instituto hacia la historia del presente a partir de la organización, entre otros, de tres seminarios: "De la guerra fría hacia el presente: América Latina en el devenir mundial", "Historia reciente de América Latina. Hechos, procesos, actores" e "Historia contemporánea y del tiempo presente en México". Paralelamente, la historia oral también forma parte de las líneas de investigación del instituto, con lo cual el vínculo con la práctica historiográfica del presente se estrechó, como muestra Garay (2007) con su trabajo en el que la historia oral se incluye como propuesta de formación de nuevas fuentes documentales no convencionales como son los testimonios orales y visuales. Es de destacar que el Instituto Mora mantiene a un grupo de investigadores registrados en este campo, lo que muestra no solo la consolidación de una línea de investigación, sino también el reconocimiento institucional como práctica historiográfica con posibilidades de avanzar en la docencia y formación de nuevos profesionales que se reconocen por sus métodos y técnicas, diferentes a los de otros campos historiográficos.

Si bien se puede afirmar que actualmente existe una comunidad de practicantes de la historia del tiempo presente o cualquiera de sus denominaciones, que se reconoce por los paradigmas y métodos específicos, al mismo tiempo existe - y es importante señalarlo- una tradición como resultado de un proceso de desarrollo 
específico de la historiografía profesional en México que siempre ha estado relacionado con las ciencias sociales para entender la sociedad contemporánea de México en el siglo XX. Este rasgo es particular pero, al mismo tiempo, producto de la evolución global de los marcos institucionales de las instituciones de educación superior y de los marcos teóricos de las disciplinas sociales y humanas que surgieron en procesos específicos que denominamos contemporaneidad en los dos periodos de posguerra mundiales.

\section{Notas}

${ }^{1}$ La primera obra de este género es la dirigida por Daniel Cosío Villegas (1955-1972). Abarca la República restaurada y el porfiriato en tres secciones: vida política -interna y externa - vida económica y vida social. Esta obra está dirigida a entender el presente, el deterioro diagnosticado por Cosío en un trabajo previo (1947) del liberalismo heredado de la República restaurada. En el lado de las ciencias sociales, el libro de Pablo González Casanova (1965) es el otro hito que anunciaba la crisis del modelo del Estado mexicano construido después de la Revolución.

2 En la década de 1980 la Benemérita Universidad Autónoma de Puebla estableció, bajo la dirección de Enrique Semo, el Centro de Estudios Contemporáneos, dependiente del Instituto de Ciencias. Contaron con una publicación, Estudios Contemporáneos; este es el único caso de un espacio institucional dedicado al ejercicio de la historia contemporánea, sin embargo, su duración fue efímera ya que no se tiene noticia de su funcionamiento al finalizar la década de su creación.

${ }^{3}$ La Escuela Nacional de Ciencias Políticas y Sociales fundada en 1951 fue transformada en Facultad de Ciencias Políticas y Sociales en 1968.

${ }^{4}$ Ver:https://es.-es.facebook.com/coloquiohistoriapresente; https://twitter.com/PresentHistoria

${ }^{5}$ Curiosamente, desde muy temprano la historia oral aparece en el horizonte historiográfico mexicano como urgencia política del rescate y conservación de los testimonios de los participantes de la revolución de 1910, cuando el INAH organizó en la Dirección de Investigaciones Históricas el Archivo Sonoro en 1959. A partir de esta instancia puede observarse una producción dedicada al rescate y resguardo del testimonio de los participantes del hechorevolucionario. Junto a ello, la historia oral se desarrolla como ejercicio historiográfico propio, con objetivos diferenciados a su encomienda inicial, al asimilar las aportaciones que las escuelas historiográficas norteamericana, francesa e inglesa realizan para la historia social, en específico la construcción de nuevos sujetos sociales al darle voz a los excluidos de la narrativa historiográfica tradicional.

${ }^{6}$ Ver: http://con-temporanea.inah.gob.mx/

${ }^{7}$ Ver: ceiich.unam.mx/0/20MunSig.php. Este programa tiene como objetivo analizar: "los principales retos y oportunidades civilizatorias que enfrenta la humanidad, en particular México y América Latina, a partir de estudiar teórica y empíricamente las dinámicas actuales del capitalismo, sus contradicciones y los procesos de resistencia social y construcción de alternativas". Lo importante del asunto es observar que la historia es una variable en una dimensión más amplia, no la única ni la más importante.

\section{Referencias}

Aróstegui, Julio (1998). "Historia y tiempo presente, un nuevo horizonte en la historiografía contemporánea”. En Cuadernos de Historia Contemporánea, 20, 15-18.

Aróstegui, Julio (2004). La historia vivida. Sobre la historia del presente. Madrid: Alianza Editorial.

Bédarida, François (1998). “Definición, método y práctica de la historia del presente". En Cuadernos de Historia Contemporánea, 20, 19-27.

Bizberg, Ilán y Lorenzo Meyer (coords.) (2003). Una historia contemporánea de México, 4 t. México: El Colegio de México/Océano.

Cordera, Rolando (coord.) (2010). Presente y perspectivas. Historia crítica de las modernizaciones en México, vol. 7. México: Fondo de Cultura Económica.

Cosío Villegas, Daniel (1947). "La crisis de México". En Cuadernos Americanos, año VI(XXXII), marzo-abril, 29-5l. Cosío Villegas, Daniel (dir.) (1955-1972). Historia moderna de México, 10 t. México: Hermes. 
Cuesta Bustillo, Josefina (1983). "La historia del tiempo presente: estado de la cuestión”. En Historia Contemporánea, 1, 227-241.

Garay, Graciela de (2007). “iPor qué estudiar la historia del tiempo presente?” En Graciela Garay (coord.), Para pensar el tiempo presente. Aproximaciones teóricometodológicas y experiencias empíricas. México: Instituto Mora.

González Casanova, Pablo (1965). La democracia en México. México: Era.

González Casanova, Pablo (coord.) (1977). América Latina. Historia de medio siglo, 2 t. México: Instituto de Investigaciones Sociales-UNAM/Siglo XXI.

Gortari Rabiela, Hira de (1990). "La historiografía contemporánea”. En Historias, 24, abril-septiembre, 45-54.

Gortari Rabiela, Hira de (2002). "El uso de conceptos y métodos en la historiografía reciente. Una aproximación”. En Pablo González Casanova (coord.), Ciencias sociales: algunos conceptos básicos. México: CEIIHC-UNAM/Siglo XXI, pp. 26-43.

Iggers, Georg G. (2012). La historiografía del siglo XX. Santiago de Chile: Fondo de Cultura Económica.

INAH (Instituto Nacional de Antropología e Historia) (2014). "Con-temporánea, nueva propuesta editorial en la red". En Boletines, 22 de agosto. Disponible en: https://inah.gob.mx/boletines/1289-con-temporaneanueva-propuesta-editorial-en-la-red.
Matute, Álvaro (1991). "Notas sobre la historiografía positivista mexicana". En Secuencia, 21, septiembrediciembre, 49-64.

Navajas Zubeldia, Carlos (2003). "El regreso de la 'verdadera historia contemporánea”. En Revista de Historia Actual, 1(1), 143-162.

Sauvage, Pierre (1998). "Una historia del tiempo presente". En Historia Crítica, 17, julio-diciembre, 59-70.

Soto Gamboa, Ángel (2004). "Historia del presente: estado de la cuestión y conceptualización". En Historia Actual Online, 3, invierno, 101-116.

Tavera Alfaro, Xavier (1955). "La carrera de Historia en México”. En Historia Mexicana, 4, abril-junio, 624-636.

Trindade, Hélgio (coord.) (2007). Las ciencias sociales en América Latina en perspectiva comparada. México: Siglo XXI.

Wallerstein, Immanuel (coord.) (1996). Abrir las ciencias sociales. México: Siglo XXI/Centro de Investigaciones Interdisciplinarias en Humanidades-UNAM.

Zavala, Silvio (1952). El Instituto Panamericano de Geografía e Historia. México: IPGH.

Zermeño, Guillermo (1997). "Notas para observar la evolución de la historiografía en México en el siglo XX". En Espacio, tiempo y forma, serie V, Historia Contemporánea, t. 10. Madrid: Facultad de Geografía e Historia-UNED, pp. 441-456.

Zermeño, Guillermo (2013). "La historiografía en México: un balance (1940-2010)”. En Historia Mexicana, LXII(4), abril-junio, 1695-1742. 